\title{
$\mathrm{M}^{\mathrm{gr}}$ Eugène Lapointe et les débuts de la grande industrie au Saguenay (1896-1930)
}

\section{Gaston Gagnon}

Volume 55, 1988

Cent cinquante ans d'histoire religieuse au Saguenay-Lac-Saint-Jean (1838-1988)

URI : https://id.erudit.org/iderudit/1006947ar

DOI : https://doi.org/10.7202/1006947ar

Aller au sommaire du numéro

Éditeur(s)

Les Éditions Historia Ecclesiæ Catholicæ Canadensis Inc.

ISSN

0318-6172 (imprimé)

1927-7067 (numérique)

Découvrir la revue

Citer cet article

Gagnon, G. (1988). $\mathrm{M}^{\mathrm{gr}}$ Eugène Lapointe et les débuts de la grande industrie au Saguenay (1896-1930). Sessions d'étude - Société canadienne d'histoire de l'Église catholique, 55, 63-74. https://doi.org/10.7202/1006947ar

Tous droits réservés @ Les Éditions Historia Ecclesiæ Catholicæ Canadensis Inc., 1988
Ce document est protégé par la loi sur le droit d'auteur. L'utilisation des services d’Érudit (y compris la reproduction) est assujettie à sa politique d'utilisation que vous pouvez consulter en ligne.

https://apropos.erudit.org/fr/usagers/politique-dutilisation/ 


\title{
Mgr Eugène Lapointe et les débuts de la grande industrie au Saguenay 1896-1930
}

\author{
Gaston GAGNON, historien
}

Répondant à l'invitation de l'organisateur de ce congrès, j'ai voulu entretenir cet auditoire de l'apport de Mgr Eugène Lapointe à l'histoire de la région et, en même temps, à celle du Québec. Certes, Mgr Lapointe n'est pas un personnage inconnu des historiens. Les articles produits par eux à son sujet sont relativement abondants, bien qu'aucune étude exhaustive sur sa vie et son œuvre n'ait été encore entreprise à partir des sources de première main contenues dans les archives.

C'est mon travail sur le patrimoine industriel de la Pulperie de Chicoutimi qui m'a amené à m'intéresser à lui. Soucieux de comprendre la réalité ouvrière à l'intérieur des moulins de pâte à papier fondés au siècle dernier par J.-E.-A. Dubuc, Mgr Lapointe, alors jeune abbé, s'est trouvé tout bonnement, si je puis dire, sur mon chemin. Voulant dépasser les études produites par Michel Têtu (1962), Robert Parisé (1978), Jacques Rouillard (1979) et Gilbert Vanasse (1986) ${ }^{1}$, j'ai instinctivement orienté ma recherche sur le dépouillement des fonds inédits conservés aux Archives nationales du Québec et aux Archives du Séminaire de Chicoutimi.

Afin d'illustrer la contribution de Mgr Lapointe à l'histoire saguenéenne et québécoise, trois aspects de sa vie ont été retenus pour les fins de cet exposé. Les deux premiers touchent la fondation de la Fédération ouvrière de Chicoutimi en 1907 et de la Fédération ouvrière mutuelle du Nord en 1912; la dernière a trait enfin à son intervention pour garder «à la

1 Michel Têtu, «La Fédération ouvrière mutuelle du nord», Relations industrielles, vol. $17, \mathrm{n}^{\circ} 4$ (octobre 1962), p. 402-421; Robert Parisé, Le fondateur du syndicalisme catholique au Québec: Mgr Eugène Lapointe. Sa pensée et son action syndicale, P.U.Q., 1978, 80p.; Jacques Rouillard, Les syndicats nationaux au Québec de 1900 à 1930, Québec, P.U.L., 1979, p. 188-191; et Gilbert Vanasse, Histoire de la Fédération des travailleurs du papier et de la forêt (CSN), Montréal, Éd. Saint-Martin, 1986, p. 19-54. 
Race» les actifs de la Compagnie de pulpe de Chicoutimi en 1924. À travers ces trois épisodes se profilent un même cheminement, une même action, soit celle de permettre aux Saguenéens et aux Québécois de se prendre en main et d'améliorer leurs conditions de vie matérielle. Ce souci de Mgr Lapointe d'incarner ses vues et ses idées dans le réel le rattache d'ailleurs à la tradition des curés Nicolas Hébert et Antoine Labelle2. Selon moi, Mgr Lapointe est loin de vouloir dominer le milieu ouvrier et accentuer le pouvoir de l'Église sur la communauté locale ou régionale; l'étude de ces documents historiques montre comment cet homme a voulu éveiller les siens à l'éducation et à l'instruction, à l'industrie et au commerce, à l'épargne et à la coopération. C'est dans cet esprit, à contre-courant je dirais du mouvement agriculturiste véhiculé à l'époque par la majorité des membres du clergé catholique ${ }^{3}$, qu'il faut situer et inscrire toute son cuvre sociale.

\section{La fondation de la Fédération ouvrière de Chicoutimi}

Selon Jacques Rouillard de l'Université de Montréal, quand l'abbé Eugène Lapointe se lance en 1904 dans l'action syndicale, il n'est pas «le premier venu dans le domaine social4». Professeur au Séminaire de Chicoutimi depuis 1882, il avait déjà été confronté, lors de ses études classiques à Québec, à des scènes brutales découlant de la misère ouvrière dans les quartiers de la «Basse-Ville». Un passage de ses Mémoires concerne justement celui de Saint-Sauveur. Un peu plus tard, de 1891 à 1893, un séjour d'études en Europe le met en contact avec cette même misère et cette même violence. Aussi, très tôt il arrive à conclure «qu'à des besoins nouveaux il fallait pourvoir par des institutions et des méthodes nouvelles ${ }^{5}$ \%.

Au même moment paraît la célèbre encyclique de Léon XIII: Rerum Novarum. Voyant dans ce texte autre chose qu'un simple document doctrinal, contrairement à la majorité des membres du clergé de son temps, il lui

2 Voir Pierre-Maurice Hébert, Le curé Hébert, 1810-1888. Un siècle d'histoire, Éditions de l'Écho, 1988; Gabriel Dusseault, Le curé Labelle. Messianisme, utopie et colonisation au Québec, 1850-1900, Montréal, Hurtubise-HMH, 1983, 392 p.

3 Sur ce mouvement, Michel Brunet, «Trois dominantes de la pensée canadiennefrançaise: l'agriculturisme, l'anti-étatisme et le messianisme», La présence anglaise et les Canadiens. Etudes sur l'histoire et la pensée des deux Canadas, Montréal, Beauchemin, 1964, p. 113-166; et pour sa contrepartie: William F. Ryan, The Clergy and Economic Growth in Quebec (1896-1914), Québec, P.U.L., 1966, 348 p.

4 Jacques Rouillard, op. cit., p. 188.

5 Michel Têtu, op. cit., p. 403; «Hommage à Mgr Lapointe, p.a., docteur en sciences sociales, économiques et politiques de l'Université de Montréal», Alma Mater, septembreoctobre 1943, p. 10; et Eugène Lapointe, Mémoires, p. 244sq., Archives nationales du Québec à Chicoutimi (ANQC), Fonds Mgr Victor Tremblay, document 813. 
vient à l'idée de le mettre en application, après avoir été reçu avec des confrères en audience privée par le pape ${ }^{6}$.

Mais avant de réaliser cet objectif, il décide d'approfondir ses connaissances sur la question ouvrière. C'est pourquoi en dehors de ses études de doctorat en philosophie, il profite de ses temps libres pour voyager, pour s'entretenir avec les gens. Car ce ne sont pas les monuments historiques qui l'intéressent, mais les échanges avec les paysans, avec les travailleurs, pour identifier les causes de leur misère et trouver des moyens d'y remédier. Comme le père Ceslas Rûtten en Belgique, il ira même jusqu'à revêtir l'habit laïc en France, où un fort mouvement anticlérical se déploie, pour faciliter ces contacts ${ }^{7}$, ce qui prouve sa très grande détermination.

En 1894, l'abbé Lapointe revient à Chicoutimi après avoir passé auparavant sept mois dans une paroisse de l'État de New York pour se familiariser avec la langue anglaise. S'adonnant à l'enseignement, il n'en poursuit pas moins son travail de documentation et de réflexion sur l'organisation ouvrière. Membre de la Société d'économie sociale de Paris fondée par Frédéric Le Play, il correspond avec plusieurs économistes et sociologues.

En 1896 naît à Chicoutimi le premier complexe de pâte à papier fondé au Canada par des Québécois. Aux yeux de l'abbé Lapointe, c'est «un commencement de conquête économique». Mais une telle initiative était vouée selon lui à l'échec, «si le travail dans les usines n'était pas libéré d'une direction étrangère omniprésente ${ }^{8}$ ", en l'occurence celle des syndicats internationaux.

L'occasion lui ayant été donnée, l'abbé Lapointe décide de mettre en pratique les enseignements de Rerum Novarum. Ami personnel du directeur-gérant des usines de Chicoutimi et partageant ses vues quant à l'avenir du Saguenay, l'abbé Lapointe se lance dans l'action. De 1903 à 1907, il sensibilise d'abord son milieu à la cause ouvrière; il tend ensuite à faire valoir auprès des plus intéressés les avantages de se regrouper, de s'associer en formant un syndicat; enfin il se met à la recherche d'une formule d'association transmissible dans des statuts et règlements qui, tout en

6 À ce sujet, voir Élie-J. Auclair, Alma Mater, vol. 1, n 7, 3 mars 1936, p. 53; O.D. Simard, «Mgr Eugène Lapointe, p.a., sa carrière», Alma Mater, Série II, vol. 1, nos 9 et 10, mai-juin 1936; Mgr Eugène Lapointe, «Les travailleurs catholiques à Rome», à l'occasion du $40^{\mathrm{e}}$ anniversaire de Rerum Novarum, Le Progrès du Saguenay, 22 mai 1931; Noël Bélanger et Jean-Guy Lalande, "Les réactions devant la montée ouvrière», dans Le collectif, Les iravailleurs québécois, 1851-1896, P.U.Q., 1973, p. 164-167.

7 Mgr Eugène Lapointe, Mémoires, p. 228 et 240.

8 Mgr Eugène Lapointe, «Les syndicats catholiques au Canada», Semaine sociale $d u$ Canada, (Québec 1921), Montréal, L’Action paroissiale, 1922, p. 376. 
s'inspirant des expériences d'ailleurs, puisse cadrer avec les aspirations et la situation des travailleurs de Chicoutimi ${ }^{9}$.

C'est ainsi qu'en collaboration avec les directeurs de la Compagnie de pulpe de Chicoutimi, dont certains recevront le titre de Commandeur de l'ordre de Saint-Grégoire-le-Grand, l'abbé Lapointe jette les bases en février 1904 d'une série de conférences sur la question ouvrière. L'évêque du diocèse, Mgr Michel-Thomas Labrecque, est le premier conférencier invité. Sa causerie sur le travail du dimanche, sujet qui deviendra un élément d'affrontement et de polémique avec Sir Willianin Price, serâ ieproduite dans le journal local, propriété d'un des directeurs de la compagnie ${ }^{10}$. En mai 1907, c'est au tour de l'abbé Lapointe de prononcer une conférence. C'est à ce moment qu'il fait part pour la première fois, publiquement, de ses idées sur la question ouvrière, qu'il souligne longuement les méfaits des syndicats internationaux:

Ces unions affiliées à l'Internationale sont désastreuses pour l'ouvrier, parce qu'elles leur enlèvent sans compensations valables, leur petite épargne, pour la déverser dans les coffres de l'Internationale, qui (supporte) avec (elle) les grèves souvent si malheureuses des grandes villes européennes et américaines.

\section{Et le conférencier de renchérir:}

On ne se fait pas d'idée des sommes fabuleuses qui sont ainsi drainées des poches de l'ouvrier canadien pour s'en aller aux États-Unis ou ailleurs, sans aucun profit pour nous. Dans la paroisse de Saint-Roch de Québec, plus de quatre-vingt mille $(80000)$ piastres sont ainsi arrachées chaque année à la population ouvrière pour s'en aller grossir le trésor de l'Internationale.

Et on se plaint que nous n'avons pas d'écoles industrielles! Que le gouvernement, sous prétexte qu'il n'en a pas le moyen ne veut pas en établir! Mais pourquoi les ouvriers n'en fondent-ils pas eux-mêmes, avec ces sommes énormes qu'ils envoient inconsidérément à l'étranger ${ }^{11}$.

Cette idée d'auto-développement, l'abbé Lapointe la répandra tout au cours de sa vie. C'est aussi dans cette même conférence qu'il dévoilera son fameux projet de former «une union purement locale» qui regrouperait les ouvriers venus de différents horizons professionnels et qui pourrait être appelée quelque chose comme la «fédération locale des ouvriers de Chicoutimi».

Invitant les quelque 250 à 300 travailleurs présents dans la salle à faire partie du comité organisateur, le mouvement qu'il lançait ce soir-là était passablement avancé, structurellement parlant, puisqu'en même temps qu'il faisait cette proposition d'association, il indiquait la création

9 Jean-Claude Drolet, «Mgr Eugène Lapointe, initiateur du syndicalisme catholique en Amérique du Nord», Saguenayensia, septembre-octobre 1966, p. 102.

10 Le Progrès du Saguenay, 8 et 11 février 1904.

11 Le Progrès du Saguenay, 16 mai 1907. 
prochaine d'une caisse d'épargne pour les ouvriers et les enfants d'ouvriers, ainsi qu'une maison destinée spécialement à leur usage, avec gymnase, salle de jeux et cabinet de lecture.

L'appel étant lancé, 175 noms vont être recueillis. Un comité restreint, formé de 25 à 30 personnes, receva le mandat de rédiger les statuts et règlements du futur syndicat ${ }^{12}$.

Oeuvre principalement de l'abbé Lapointe, de son neveu l'avocat Simon Lapointe et du protonotaire F.-X. Gosselin, la constitution fera de la Fédération une union d'étude consacrée à la création, au développement et à la direction d'œuvres ouvrières de toutes sortes. Excluant le syndicat de l'influence des partis politiques et de celles des compagnies et des patrons, les statuts et règlements n'en comprenaient pas moins, selon l'article 8 , la présence de «membres honoraires».

Pour l'abbé Lapointe, cette présence était fondamentale; elle assurait au syndicat un essor plus rapide dans la société. Cette question, qui sera une des principales causes du demi-échec de la Fédération, ne fera pas l'unanimité parmi les membres du comité organisateur.

Se basant sur leur vécu, certains membres, dont le notaire MauriceOvide Bossé, qui avait participé à Lowell à la formation d'un syndicat ouvrier, soutiendront «qu'il ne [pouvait] y avoir à Chicoutimi de société vraiment ouvrière que celle composée uniquement [de travailleurs]». Selon ces dissidents, c'était de plus «une marque de non-confiance dans l'intelligence et dans l'habileté [des ouvriers]» que d'accepter parmi eux des membres de l'extérieur qui pouvaient non seulement faire partie du conseil d'administration mais qui étaient habilités également à voter sur les décisions ${ }^{13}$.

Malgré ces objections, l'abbé Lapointe convoquera pour le 24 novembre (1907) la réunion de fondation de la Fédération. Les journaux locaux, Le Progrès du Saguenay et Le Travailleur, publiciseront l'événement. À la mi-décembre, une cinquantaine de jeunes travailleurs adhéraient au mouvement dont la cotisation mensuelle s'élevait alors à 25 cents.

À partir de 1908, l'abbé Eugène Lapointe délaisse la «direction» du syndicat pour se consacrer plus spécialement à la mise sur pied d'une

12 ANQC, Fonds Mgr Victor Tremblay, document 359: lettre de l'abbé Eugène Lapointe à Joseph Girard, 11 décembre 1907; ANQC, Fonds des syndicats nationaux: Statuts et règlements de la Fédération ouvrière de Chicoutimi, article 2, p. 1-2.

13 ANQC, Fonds des syndicats catholiques, s.c.: Lettre de Maurice-Ovide Bossé, N.P., à l'abbé Eugène Lapointe, 14 décembre 1907; ANQC, Fonds Mgr Victor Tremblay, document 359-F: lettre de Joseph Girard à l'abbé Eugène Lapointe, 5 décembre 1907; et au sujet de l'article 8, voir Statuts et règlements de la FOC, p. 5-6. 
école du soir pour les ouvriers, à la fondation de la Ligue de tempérance, de la Caisse de petite économie ainsi que de la Caisse populaire de Chicoutimi. Créée en 1911, cinq ans après celle de Lévis, la Caisse de Chicoutimi visait à favoriser la pratique de l'épargne et à répondre au besoin de crédit de ses membres. Les institutions bancaires existantes, ayant été conçues d'abord et avant tout pour les classes industrielle et commerciale, il était en effet impossible pour un ouvrier ou un cultivateur, autrement qu'en recourant au prêt usuraire, d'avoir accès à des prêts personnels ou hypothécaires. À une époque où la construction domiciliaire, était en plein progrès dans la ville, les facilités de crédit devenaient tout aussi importantes pour le travailleur de la «pulpe» que le regroupement syndical. C'est pourquoi l'abbé Lapointe se préoccupe de cette question et accepte de siéger comme administrateur de la Caisse, à côté, du reste, des cadres supérieurs de la Compagnie de pulpe de Chicoutimi ${ }^{14}$.

Excluant les problèmes à caractère professionnel, la Fédération ouvrière de Chicoutimi n'obtiendra pas, il faut bien le dire, toute la faveur escomptée par ses organisateurs. Du côté de son fonctionnement, la même remarque pcut s'appliquer car, durant ses trois ans et demi d'existence (déc. 1907-avril 1911), seulement 20 réunions du conseil et 10 assemblées générales eurent lieu ${ }^{15}$.

Plusieurs raisons peuvent être invoquées pour justifier cette abstention ou cette indifférence. Parmi elles, on peut citer la crainte de représailles de la part de l'employeur, l'esprit individualiste, le refus de voir le prêtre se mêler des questions sociales, la méfiance à l'endroit de l'abbé Lapointe lié d'amitié avec Dubuc, de même que le caractère par trop idéaliste de certains articles des statuts et règlements de la Fédération, en particulier de l'article $8^{16}$.

Les avantages qu'elle procurait n'étant pas «assez apparents pour frapper les esprits et soutenir [la] confiance», la FOC n'avait pu en somme se maintenir en activité qu'avec un nombre très restreint de membres, une «élite» convaincue, envers et contre tous, du bien-fondé de l'œuvre ${ }^{17}$. Aussi en 1912, à la suite d'un voyage d'études en Belgique et en Francel${ }^{18}$

14 L'Écho paroissial du Sacré-Caur, novembre 1911, p. 160-161; Archives de l'Évêché de Chicoutimi (AEC), «Liste des officiers de l'Action populaire économique», série 9, cote 35, vol. 25, pièce 2-b; et Yves Roby, Alphonse Desjardins et les Caisses populaires, 18541920, Montréal, Fides, 1964, p. 36.

15 ANQC, Fonds Mgr Victor Tremblay, document 718: Livre des procès-verbaux de la FOC, séances du 10 avril et 13 septembre 1908, et du 20 décembre 1909, p. 3-72.

16 Mgr Eugène Lapointe, «Les syndicats catholiques au Canada», p. 376; Jean-Claude Drolet, op. cit., p. 105.

17 Mgr Eugène Lapointe, op. cit.

18 Sur ce voyage où il rencontrera $M$. Mousset, secrétaire-général des œuvres sociales de Bruxelles et l'abbé Woessen, Le Progrès du Saguenay, 26 mars 1908, et Le Travailleur, 26 mars 1908. 
et après avoir été affecté à temps plein par son évêque aux œuvres sociales dans le diocèse, l'abbé Lapointe entreprend de modifier la structure de la Fédération.

Élaborant une nouvelle constitution en rayant les éléments qui faisaient ombrage à son œuvre, il jette les bases de la Fédération ouvrière mutuelle du Nord (FOMN) dont une des sections deviendra, à sa dissolution en 1923, le Syndicat $n^{\circ} 2$ de la pulpe et du papier. Et c'est vraiment cette initiative qui laissera sa marque dans la population ouvrière et dans l'historiographie du monde syndical ${ }^{19}$.

\section{La fédération ouvrière mutuelle du nord}

L'engouement déployé à l'endroit de la FOMN, qui par certains de ses aspects avait l'allure d'une société secrète, chacun des syndiqués devant prononcer un mot de passe pour pouvoir pénétrer dans la salle d'assemblée ${ }^{20}$, est attribuable en grande partie à l'opiniâtreté de son fondateur. Pendant deux ans, l'abbé Lapointe ne ménage aucun effort pour développer la nouvelle Fédération. Il aménagera même un local au beau milieu du quartier ouvrier afin de recevoir les travailleurs venus le rencontrer pour discuter, se renseigner et obtenir des conseils et des services de tous genres. «Par son dévouement, écrira Michel Têtu, il [arrivera], peu à peu, à détruire les préjugés profondément enracinés chez les ouvriers, et à les gagner à la Fédération ouvrière ${ }^{21}$.»

D'autre part, cette popularité s'explique par le fait que l'Internationale, inquiète du succès de la Fédération, décidera de lui faire la guerre en entreprenant une campagne de maraudage et en essayant de convaincre les ouvriers indécis de joindre ses rangs. Or, c'est justement le contraire qui arriva, l'abbé Lapointe participant aux assemblées contradictoires et convainquant les travailleurs d'adhérer à son mouvement. Résultat: avec le support de la Compagnie de pulpe de Chicoutimi et de sa dizaine de sociétés subsidiaires qui reconnaîtront la FOMN en août 1912, le nombre de membres ne cessera d'augmenter. De 421 à la fin de l'année 1912, il s'élè-

19 Mgr Eugène Lapointe, op. cit., p. 377; Robert Parisé, op. cit., p. 51-52; et AEC, Registres, série B; actes, vol. II, p. 329-330: lettre de Mgr M.-T. Labrecque à A.S. McNichols, 29 août 1926.

20 À ce sujet, voir l'entrevue de Gaston Gagnon avec Adjutor Lévesque, 4 mars 1983, Centre d'interprétation de la pulperie de Chicoutimi: projet d'enquêtes orales; ANQC, Fonds Mgr Victor Trembiay, document 921: Livre des procès-verbaux de la FOMN, 16 janvier 1916, p. 24, 19 mai 1916, p. 44 et 2 avril 1917, p. 64; et ANQC, Fonds des syndicats catholiques: Rapport du comité des griefs, 23 janvier 1927.

21 Michel Têtu, op. cit., p. 416. 
vera à près de 1100 en juin 1914, soit une augmentation de plus de $60 \%$ en l'espace d'un an et demi22.

Créée officiellement en mars 1912, à la suite de la publication de la lettre pastorale de Mgr Michel-Thomas Labrecque, et dotée d'une charte civile, la Fédération ouvrière sera dans un premier temps une société de secours mutuels et de prévoyance et ce, à l'exemple des modèles européens. C'est ainsi qu'entre 1912 et 1916, en raison de la force du pouvoir d'achat de ses membres, une coopérative de consommation sera formée en

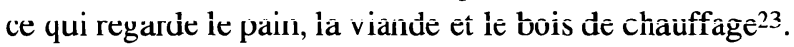

Mais, outre ces éléments qui se réfèrent à la mutualité, ce qui caractérisera la FOMN et le Syndicat $n^{\circ} 2$ de la pulpe et du papier, ce sera l'accent mis sur les questions d'ordre professionnel, contrairement à la FOC. Dans la mesure où celles-ci rejoignent directement l'ouvrier à l'intérieur de son milieu de travail, qu'elles renvoient aussi bien aux conditions salariales qu'aux conditions de santé et de sécurité, ces questions sont de première importance pour qui veut mesurer et analyser l'écart existant entre le discours patronal ouvrier et le vécu quotidien des travailleurs de la «pulpe».

Or, en dépit de la philosophie paternaliste pratiquée par Dubuc et par les autres membres du conseil d'administration de la compagnie ${ }^{24}$, la distance était considérable. Certes, d'un point de vue fonctionnel et architectural, les moulins étaient parmi les plus remarquables du Québec; les conditions de santé et de sécurité n'étaient ni pires ni meilleures que dans les autres usines, technologie oblige. Toutefois, en ce qui concerne les conditions salariales, la différence était non seulement considérable mais les payes en souffrance, symptôme d'un problème de fonds de roulement et de gestion, rendront petit à petit la situation intolérable. En effet, ce qui pouvait paraitre en 1906 un épiphénomène, un accident de parcours à caractère administratif, va se convertir en véritable «système» échelonné sur plusieurs semaines, voire sur plusieurs mois en 1912, 1914, 1915 et 1922. De plus, les travailleurs de Chicoutimi, comparés aux autres membres de l'industrie, se classent au-dessous de la moyenne provinciale au chapitre de l'échelle salariale. Et ce double constat est d'autant plus surprenant que l'on se trouve à une période où la compagnie est en plein essor, tant du côté de ses constructions que du côté de sa production, et

22 Le Progrès du Saguenay, 5 juin, 2, 9 et 10 octobre 1913; ANQC, Fonds Mgr Victor Tremblay, document 718,4 août 1912 , p. 104, et pour les éléments statistiques voir p. 78291.

23 ANQC, Fonds Mgr Victor Tremblay, document 921: Livre des procès-verbaux de la FOMN, séance du 11 août 1912, du 29 février, du 10 et 22 avril 1916, et du 23 février 1919.

${ }^{24}$ Gaston Gagnon, op. cit., p. 80-86. 
que ses profits enregistrent des percées à la hausse impressionnantes, passant de 160000 \$ en 1913 à plus de 2 millions \$ en 192025.

Dans ces conditions, on comprendra les prises de position de la FOMN qui, à maintes reprises durant ces années, cherchera à faire corriger la situation et s'opposera à tout projet de diminution de salaires de la part de l'employeur, en 1914, en 1922 et en 192426. En 1914, en particulier, la tension sera à ce point élevée entre le syndicat et la compagnie que l'abbé Lapointe, devenu Mgr Lapointe, par suite de sa promotion au rang de Protonotaire apostolique, sera accusé, en pleine assemblée syndicale, d'avoir trompé les travailleurs. Un membre du syndicat affirmera à cette occasion que la compagnie trouve facilement de l'argent quand il s'agit de payer les entrepreneurs forestiers ou d'acheter le moulin de pulpe de ValJalbert, mais qu'il en est autrement lorsqu'elle doit faire face à ses engagements à l'endroit de ses employés ${ }^{27}$.

Mgr Lapointe désapprouva publiquement l'attitude de la compagnie. Il rappellera aux ouvriers réunis en assemblée spéciale le 22 mars 1914 son effort mené auprès de Dubuc afin de rétablir la situation. «Si vous voulez faire la grève, leur dira-t-il, vous avez beau, je ne vous [empêcherai] pas [car], c'est votre droit [d'être] payé28.»

Incidemment, ce ne sera pas la seule intervention de cette nature que devra entreprendre Mgr Lapointe. En mai 1918, à la suite de plusieurs demandes d'augmentation de salaire de la part du syndicat, celui-ci écrira:

Je suppose que vous savez que vos ouvriers sont depuis des mois déjà dans un état de quasi exaspération. Ils ont l'impression fâcheuse d'être une faiblesse exploitée par une force. Ils sentent bien que tant qu'ils n'auront pas décidé de renoncer à vivre dans leur Chicoutimi, ils sont à la merci d'un patron sans concurrent.

La cause de cet état d'esprit [...] est multiple. C'est d'abord le règlement arbitraire de plusieurs difficultés qui ont surgi depuis l'hiver dernier; ensuite, les gages: il est notoire que les ouvriers de Chicoutimi n'ont pas les gages de ceux de Kénogami, de Grand'Mère, Chawinigan [sic], La Tuque et le Cap de la

25 Le Travailleur, 9 août 1906 et Le Progrès du Saguenay, 29 mars 1906, 25 juin 1914, 23 septembre 1920 et 31 mars 1921; ANQC, Fonds Mgr Victor Tremblay, document 718: séances des 4 et 28 juillet 1912, séance du 22 mars 1914; document 921: Livre des procèsverbaux de la FOMN, séance du 31 octobre 1915 et séance du 4 juin 1922; et ANQC, Fonds Dubuc, APG-1.76.1.14: lettre d'Antoine Dubuc à son père, 30 mars 1919.

26 ANQC, Fonds Mgr Victor Tremblay, document 718, séance du 30 juin 1914; ANQC, Fonds des syndicats catholiques, résolution du 28 janvier 1922; aussi lettre du ler février 1924 dans le même fonds. À noter que cette pratique sera très répandue au Québec durant la période d'après-guerre, ce qui ne manquera pas de susciter la radicalisation du mouvement syndical et le déclenchement de piusieurs grèves, Jacques Rouillard, op. cit., p. 243-244sq.

27 ANQC, Fonds Mgr Victor Tremblay, document 718: séance du 15 mars 1914, p. 249252.

28 Ibid, p. 263. 
Madeleine. Pourtant, la vie coûte plus cher ici que dans ces localités; prenez seulement la lumière, l' eau et les taxes, vous en aurez la preule.

En présence de cette initiative déplorable comptent-ils sur la Fédération ouvrière pour les aider? Un mot qu'ils ont trouvé expriment [sic] bien leur pensée à ce sujet: ils l'appellent la "Figuration ouvrière". C'est dire qu'ils sont loin de se faire illusion sur le poids de leur organisation ouvrière.

[...] Résultats pratiques: malgré certaines apparences, c'est dans C'hicoutimi que l'unionisme catholique est le moins ancré [...] [Et comme] cette lettre doit avoir une conclusion pratique: la voici: il est grand temps que les ourriers aient une !égitime augmentation de gages. Il leur faut vivre avant tout, et cette lacune comblée leur fera oublier bien des choses 29 .

$\mathrm{Si}$ explicite et convainquante que soit cette lettre, la compagnie ne consentira à accorder une augmentation de $15 \%$ à ses travailleurs qu'en 1920. Et malgré cette amélioration, les employés de Chicoutimi ne pourront que difficilement faire face à leurs obligations, le coût de la vie étant reconnu comme très élevé dans la ville. Alors que celui-ci était établi à $35 \$$ par semaine en 1924 par le syndicat, les ouvriers ne toucheront que $20 \$$ ou $27 \$$ pour leurs gages 30 .

Avec de tels salaires qui pouvaient atteindre autour de 400 ou $500 \$$ annuellement contrairement aux professionnels dont les honoraires pouvaient dépasser les $5000 \$$, on peut comprendre le caractère particulier du quartier du «bassin», le quartier ouvrier, qui, avec ses maisonnettes en bois et son allure dépouillée, était perçu par les autres habitants pour être une véritable «ville dans la ville».

Certes, la Compagnie de pulpe de Chicoutimi ainsi que son réseau de sociétés subsidiaires avaient contribué considérablement à la modernisation et au développement du Saguenay et du Lac-Saint-Jean, mais la structure de ces entreprises était basée, elle aussi, sur la notion de profit. C'est pourquoi, loin d'être dupes de leur situation, les travailleurs de la Fédération se montreront davantage revendicateurs d'année en année. Si la faillite du principal client de la Compagnie de pulpe, la Becker and Co. de Londres, peut expliquer en partie la banqueroute de Dubuc et de son groupe en 1924, l'entreprise se trouvait également minée de l'intérieur par suite de la dégradation de ses rapports avec ses ouvriers, ceux-ci ne désirant plus être laissés pour compte ${ }^{31}$. Mais avec la mise en faillite, il n'était plus question de s'aventurer dans ce genre de combat. Pour Mgr Lapointe et pour les autres aumôniers du syndicat, il fallait veiller à maintenir les emplois à l'intérieur de la nouvelle organisation et, surtout, travailler à ce que l'entreprise demeure aux mains des Canadiens français.

29 ANQC, Fonds Mgr Victor Tremblay, document 723-T.

30 Voir ANQC, Fonds Mgr Victor Tremblay, document 723-T: lettre de Mgr Eugène Lapointe à J.-E.-A. Dubuc, 20 mai 1918; et Le Progrès du Saguenay, 21 avril 1921.

31 Gaston Gagnon, op. cit., p. 137-156. 


\section{La banqueroute de la Compagnie de pulpe de Chicoutimi}

Soucieux de voir les siens occuper les postes qui leur reviennent dans l'industrie, dans la finance et dans l'économie du pays, Mgr Lapointe sera effectivement l'un des premiers à se préoccuper de l'avenir des actifs de la Compagnie de pulpe de Chicoutimi. Quelques jours à peine après la mise en faillite de l'entreprise de Dubuc, il écrit à Tancrède Bienvenue, viceprésident et directeur général de la Banque provinciale du Canada, pour lui exposer son point de vue sur cette affaire:

S'il s'agissait d'une affaire anglaise, soutiendra-t-il, mes compatriotes anglo-canadiens auraient trouvé déjà une solution, et avec moins de bruit; que si M. Dubuc était anglais, ses compatriotes auraient trouvé moyen de le tirer d'embarras et n'auraient pas songé à livrer à n' importe quel prix une propriété de cette valeur à des Canadiens français.

La région du Saguenay et du Lac-Saint-Jean est une petite province. [...] Or, la Cie de Pulpe, par sa situation stratégique, tient la clef de cette région. Ce fut le coup de maître de Dubuc de s'emparer de cette clef. Il a vu de haut et de loin. On peut penser qu' il a beaucoup embrassé, trop pour ses moyens. Mais c'est fait. Il ne tient qu'à nous maintenant de garder à la race [...] cette incomparable propriété. La laissera-t-on aller? Laissera-t-on tomber en des mains étrangères le fruit du travail de trente ans de l' un des nôtres, qui, somme toute, nous fait le plus d'honneur? Dans la grande industrie de la pulpe, c'est à peu près tout ce que nous avons de vraiment important.

\section{Et Mgr Lapointe de continuer:}

[...] L'Ottawa, le St-Maurice, sont aux Anglais et aux Américains. Vous savez ce que va devenir avant longtemps le St-Maurice. Il reste le Saguenay. C'est la seule réserve forestière vraiment importante de la Province. Si on tient compte de ses sources inépuisables d'énergie électrique et de son port océanique, le Saguenay [...] est appelé à devenir l'un des centres industriels les plus importants de la province de Québec, c'est incontestable.

Pourquoi alors abandonnerions-nous si légèrement tout cela à des Anglais?

$N^{\prime}$ est-il pas temps, cher Monsieur, que dans les affaires, dans la finance, on tienne un certain compte de ce que nous sommes et de ce que nous devons être ${ }^{32}$ ?

Ce «discours» teinté de nationalisme ne ressemble-t-il pas à celui des patriotes de 1837-1838? En tous les cas, il s'inscrit à l'intérieur d'une tradition que Mgr Lapointe connaissait et qu'il avait propagée, en particulier à l'intérieur des murs du Séminaire de Chicoutimi.

En novembre 1925, au moment où le comité des créanciers de la Compagnie de pulpe présidé par Albert Stewart McNichols, un ancien directeur-gérant de la célèbre maison de courtage L.-G. Beaubien de Montréal, enclenche le processus de liquidation des actifs, Mgr Lapointe

32 ANQC, Fonds Mgr Victor Tremblay, document 728-I: lettre de Mgr Eugène Lapointe à Tancrède Bienvenu, 19 mars 1924. 
ne peut s'empêcher de poursuivre son combat. Cette fois c'est au banquier Joseph Versailles qu'il s'adresse:

Nous n'avons jamais voulu croire à la parole d'un ancien premier ministre [il voulait parler de Lomer Gouin] prononcée ici même: que «les Canadiens-français sont destinés à n'être toujours que des scieurs de bois et des porteurs d'eau», et nous avons lutté avec l'énergie du désespoir.

Serons-nous vaincus enfin? Oui ou non, dans ce Saguenay, incomparable joyau, que nous espérions garder à la race, ne serons-nous vraiment à la fin, que des bûcherons? Nous y allons - je posais la question à D. [Dubuc] hier soir. Les yeux iui rouiaient dans i" eau - .

Et Versailles? repris-je! [...] À son insu, je vous en donne ma parole d'honneur, je vous pose ce matin la même question: et Versailles ${ }^{33}$ ?

On connait la suite, la prise de contrôle de la Price Brothers Company et de la Port Alfred Pulp and Paper, l'implantation de la compagnie Alcan au Saguenay-Lac-Saint-Jean, trois entreprises qui appuieront leur développement à partir de l'héritage laissé par Dubuc et par son groupe ${ }^{34}$. Le rêve d'une "province dans la province» venait de prendre fin. Une autre époque venait de sonner, celle des grands barrages hydro-électriques et des nouveaux noyaux industriels du type d'Arvida, de Riverbend et d'île Maligne.

Mgr Lapointe ne restera pas indifférent face à ces projets. Pour lui, ils s'inscrivaient directement à l'intérieur d'un régime colonial que les politiciens avaient contribué à perpétuer. Gardant toutefois confiance dans l'avenir, il écrira à la fin de ses Mémoires: «Notre libération viendra mais il faudra le temps avec une éducation appropriée ${ }^{35}$.» Ce message d'espoir, fondé sur notre éveil national et sur l'éducation du plus grand nombre, ne résume-t-il précisément pas tout ce qu'à été au fond la vie et l'œuvre du premier fondateur du syndicalisme catholique au Québec?

33 ANQC, Fonds Mgr Victor Tremblay, document 728-M: lettre de Mgr Eugène Lapointe à Joseph Versailles, 19 novembre 1925.

34 Sur l'implantation de la grande industrie au Saguenay-Lac-Saint-Jean, voir Gaston Gagnon, Un pays neuf. Le Saguenay-Lac-Saint-Jean en évolution, Alma, Éditions du Royaume, 1988, p. 123-161.

35 Mgr Eugène Lapointe, Mémoires, extrait reproduit dans Saguenayensia, vol. 22, nos 3-4, (mai-août 1980), p. 127. 